\title{
Jean Le Blond, premier traducteur français de l'Utopie
}

L'objectif de cet article est de mettre en évidence les buts personnels poursuivis par Jean Le Blond en traduisant du latin en français un livre aussi original et atypique que l'Utopie de Thomas More. Nous souhaitons de la sorte contribuer à une meilleure compréhension de la réception de l'œuvre de More auprès d'un public moins privilégié que celui des grands humanistes traditionnellement retenus par l'histoire littéraire.

Pour étudier cette question, il convient tout d'abord de bien connaitre le traducteur. En effet, sa vie et ses prises de position antérieures à la traduction de l'Utopie (publiée en 1550) éclairent notablement la courte préface qu'il joint à 1'ouvrage. Le Blond est généralement méconnu. Les études à son propos sont fort peu nombreuses et parfois imprécises. Pour répondre à notre interrogation première, et étant donné le peu d'informations contenues dans la préface laissée par Le Blond, un bilan des acquis devait être dressé, afin d'avancer des hypothèses autorisées concernant notre question. Un retour aux textes était nécessaire. À partir de ce moment-là seulement, il devenait possible d'examiner la première traduction française de l'Utopie.

\section{Jean Le Blond}

Le Blond a pris une part active à la vie de son époque. Avocat, curé, poète, défenseur de la langue française, ses rôles furent multiples. Il prit part aux débats concernant quelques grands événements de son époque étudiés par l'histoire littéraire, notamment la querelle de Marot et Sagon ou la défense de la langue française. Le propos de cet article n'est pas de résumer ces différents sujets de recherche, mais d'inscrire Le Blond dans la dynamique de ces problématiques, et d'ainsi mieux comprendre sa position et ses prises de position dans l'espace littéraire de l'époque. 


\section{La réception par la critique}

Pour connaître les dispositions de Le Blond, une mise au point biographique doit être envisagée. Les articles existant à ce sujet se copient abondamment l'un l'autre. Il faut dire que Le Blond ne bénéficie pas d'une image très attirante; les critiques sont en effet unanimes : retardé notoire, attaquant la gloire nationale qu'est Clément Marot, fermé aux idées nouvelles du temps, il est condamné sans appel.

La campagne de dénigrement commence avec l'abbé Goujet en 1747, dans son ouvrage la Bibliothèque françoise, ${ }^{1}$ dont les propos seront repris par Du Verdier et La Croix du Maine. ${ }^{2}$ Le Blond est présenté comme un concurrent déclaré de Clément Marot auquel celui-ci ne daignera même pas répondre. Deux épîtres du Printemps de l'humble espérant ${ }^{3}$ dans lesquelles Marot est mis en cause sont signées de noms différents (Jean Le Blond et le général Chambor), ce qui n'émeut en aucune manière l'abbé Goujet. Forgé à partir de présomptions sans fondement, de sous-entendus et d'a priori déplacés, l'avis de Goujet ne sera mis en doute qu'à partir de la fin des années 1950, par Mayer surtout. Mais il laisse des traces importantes dans les histoires littéraires et les monographies. ${ }^{4}$

Il faut attendre C.-A. Mayer pour que l'on s'interroge réellement sur le rôle de Le Blond dans cette querelle. Mayer, en étudiant la religion de Clément Marot, s'attarde en détail sur les deux épîtres, ${ }^{5}$ et conclut à propos de celle de Le Blond :

Il ne s'agit donc ni d'inimitié personnelle, ni de jalousie. Ce qui a déterminé Leblond à prendre la plume, ce sont les vers de l'épître au roi, où le poète s'élève avec éloquence contre la persécution des luthériens. Aussi la réponse de Leblond à cette protestation estelle le passage le plus violent du poème. ${ }^{6}$

1 Abbé Gouset, «Jean Le Blond », dans Bibliothèque françoyse. Paris, P.-J. Mariette, 1747, t. 11, pp. 106-112. Rééd. : C.-P. GouJET, Bibliothèque françoise ou histoire de la littérature française. Genève, Slatkine Reprints, 1966, vol. 2 (t. 7-12), pp. 515-516.

2 Antoine Du Verdier (Sieur de Vauprivas) et François de LA Croix du Maine, « Jean Le Blond », dans Les Bibliothèques fiançoises. Éd. par Jean-Antoine RigoLEY DE JuvionY, Paris, Saillant et Nyon, 1772-1773, t. 1 , p. 452. Rééd. : Graz, Akademische Druk und Verlagsanstalt, 1969.

3 Jean LE BLOND, Le Printemps de l'humble espérant. Paris, Arnoul Langelier, 1536.

4 Voir P. BonneFon, «Le différend de Marot et de Sagon », dans Revue d'histoire littéraire de la France, 1894, pp. 103-138 et 259-285. - G. DuBosc, «Introduction », dans Querelle de Marot et Sagon. Éd. par É. PIcoT et P. Lacombe. Rouen, Imprimerie A. Lainé, 1920. Rééd. : Genève, Slatkine Reprints, 1969. - H. GuY, Histoire de la poésie fiançaise du XVI' siècle. Tome II : Clément Marot et son école. Paris, Honoré Champion, 1926 (rééd. en 1928). - C. Kinch, La poésie satirique de Clément Marot. Paris, Boivin \& Cie, 1940. Réed. : Genève, Slatkine Reprints, 1969. Voir aussi Y. Giraud et M.-R. Jung, La littérature française. La Renaissance 1 : 1480-1548. Paris, Arthaud, 1972. Le lecteur verra à quel point ces opinions sont dépassées.

5 Jean LE BLOND, «Epistre à Clement Marot responsive de celle parquoy il se pensoyt purger d'heresie lutheriane ». Éditée par C.-A. MAYER dans La religion de Clément Marot. Genève, Droz, 1960, t. 29, pp. 165-170. TravauX d'Hunanisme ET De Renaissance. - Jean Le Blond, « Epistre du general Chambor responsive à l'epystre de Clement Marot qu'il envoya au Roy treschrestien françoys ». Éditée par C.-A. MAYER dans «Clément Marot et le Général de Caen », dans Bibliothèque d'Humunisme et Renaissance, 1958, t. 20, pp. 278-287.

6 C.-A. MAYER, La religion de Clément Marot, op. cit., p. 79.
L'analyse du poème permet de mettre en évidence qu'à l'exception de ce passage où les insultes fleurissent, le reste du texte se présente comme un argumentaire construit.

Mayer a également analysé le poème du général Chambor, qu'il différencie sans discussion possible de Le Blond. Il compare leurs deux « epistres responsives »; plusieurs arguments sont contradictoires, et le style les oppose également.

La dernière étape à propos de la critique de Le Blond est constituée par un colloque édité par G. Defaux autour de la génération Marot. M. Simonin y a consacré sa communication. ${ }^{7}$ Il y opère une mise au point bibliographique de et à propos de l'auteur et souligne combien l'affaire Marot ne constitue qu'une goutte d'eau dans une production essentiellement tournée vers la traduction et la langue française. La génération poétique suivante, celle de Ronsard et $\mathrm{Du}$ Bellay, s'était pourtant déjà arrêtée à l'épître contre Marot, négligeant la proximité de certaines idées des textes de Le Blond avec les leurs à propos de la défense du français.

À défaut de succès pour sa poésie engagée, Le Blond aurait pu voir sa réputation postérieure sauvée par sa traduction de l'Utopie. Sur ce point, il joua également de malchance. Neuf ans après sa parution en 1550, l'œuvre fut réimprimée chez Jean Saugrain à Lyon, sans que son nom ne soit mentionné. Seule subsiste la devise de Le Blond, «Espoir en mieux ». Cette seconde édition, due à Barthélemy Aneau, porte même la mention «traduite nouvellement de latin en françoys ». On peut affirmer, à la suite de B. Hosington, que de telles pratiques étaient courantes à une époque où les droits des auteurs et des imprimeurs comptaient peu. Mais les conséquences pour la reconnaissance de l'auteur de la première traduction française de l'Utopie furent importantes :

[...] the confusion surrounding the authorship of the translation persisted from $\mathrm{Du}$ Verdier on through Père Niceron, who even attributes the 1550 edition to Aneau, as do several Aneau's biographers. Even the catalogues of the Bibliothèque nationale and the several Aneau's biographers. Even the catalogues of
British Library enter the two editions under Aneau. ${ }^{8}$

Une vérification dans le catalogue de la Bibliothèque nationale confirme cette dernière affirmation : Aneau apparaît bien dans la notice bibliographique de l'édition de 1550, sous la rubrique «Autre(s) auteur(s)», avant Jean Le Blond, alors qu'il n'a rien à y faire. Cette confusion fut clarifiée ces dernières années, ${ }^{9}$ mais de nombreux ouvrages de référence antérieurs ${ }^{10}$ commettent cette erreur.

7 M. Simonin, « À chacun son fréron : Jean Le Blond, adversaire (?) de Clément Marot », dans La génération Marot. Poètes français et néo-latins (1515-1550). Actes du colloque international de Baltimore. Édités par G. Marot. Poetes français et néo-latins (1515-1550). A
Defaux. Paris, Honoré Champion, 1997, pp. 405-424.

8 B. Hosingron, «Early French Translations of Thomas More's Utopia : 1550-1730 », dans Humanistica Lovaniensia, t. 33, 1984, p. 119.

${ }^{9}$ Voir, en 2000, le catalogue de l'exposition de la Bibliothèque nationale intitulée Utopie. La quête de la société idéale en Occident, organisée en collaboration avec la Bibliothèque de New York, qui identifie clairement le premier traducteur de l'Utopie. L. T. SARGent et R. Schaer, Utopie. La quête de la société idéale en Occident. Paris, Bibliothèque nationale de France - Fayard, 2000, p. 115.

10 Voir par exemple A. Cioranescu, L'avenir du passé. Utopie et littérature. Paris, Gallimard, 1972, p. 120. 


\section{Éléments biographiques}

Peu de renseignements existent sur la vie de Jean Le Blond. La plupart des indices biographiques relevés par M. Simonin viennent de ses œuvres, d'un accès difficile pour la plupart. ${ }^{11}$ Nous nous bornerons à étudier celles qui servent notre propos, permettent de mieux comprendre la personnalité de l'auteur et de l'inscrire dans un contexte historique et social bien défini. Comme précédemment signalé, deux manuscrits conservés à la Bibliothèque nationale contiennent des renseignements biographiques : l'un est un recueil de chants royaux, ballades et rondeaux en l'honneur de la Vierge, 12 l'autre est une chronique de Normandie rédigée par Le Blond. ${ }^{13}$ Le premier mentionne sa fonction, avocat au Parlement de Normandie, qui accompagne sa signature au bas d'un poème (Jo. Le Blond, « curie parlamenti advocatus »). ${ }^{14}$ Le second, autographe, comporte au folio 529 barré 532 un poème de Le Blond, déjà cité par G. Charlier, ${ }^{15}$ l'un des seuls critiques modernes à avoir manifesté un intérêt pour Le Blond ; il s'agit de distiques latins traduits par Le Blond lui-même.

Barra mihi patria est, frugibus bene commoda, quae bis

Millibus Ebroica distat ab urbe novem

Erudiit puerum me docta Lutetia ; quinque

Nos codices gallo fecimus ore loqui :

Neustria tum demum nostro instaurata labore,

Donetur soli gloria tota Deo.

La Barre est mon pays, lieu beau et fructueux

Esloigné seulement de neuf lieues d'Évreux.

Paris la docte $m$ 'a en mon enfance instruict.

De latin en françois j'ay cinq livres traduict.

En mon dernier labeur je recoeuilly l'hystoire

De nostre Normandie. A Dieu en soit la gloire !

La traduction de l'Utopie est signée « Iehan le Blond d'Evreux »;16 différentes pièces du Printemps de l'humble espérant, principal recueil de Le Blond paru en 1536, concernent également cette ville. À un peu moins de quarante-cinq kilomètres de celle-ci, se trouve la Barre-en-Ouche, localité dont semble provenir notre auteur.

11 Pour une liste complète des œuvres de Le Blond, voir M. SIMONIN, op. cit., pp. 421-424.

12 Chants royaux, ballades et rondeaux du pays de Normandie, B.N.F., Ms. Fonds fr. 24408 . On trouve une brève description de ce manuscrit dans le catalogue de la B.N.F. et chez G. Gros, Le poète, la vierge et le prince du Puy. Étude sur les puys marials de la France du Nord du XIV' siècle à la Renaissance. Paris, Klincksieck, 1992, p. 234. SAPIENCE.

13 Jean Le BLond, Histoire de Normandie. B.N.F., Ms. Fonds fr. 18931

14 B.N.F., Ms. Fonds fr. 24408 , fol. $71 r^{\circ}$.

15 G. ChartirR, «Jean Le Blond et son apologie de la langue française (1546)», dans Revue de l'Instruction publique en Belgique, n" 55, 1912, pp. 331-344.

16 Th. MORE, La description de l'isle d'Utopie. Trad. par Jean LE BLOND. Paris, Charles l'Angelier, 1550.
Une pièce restée inédite à ce jour, connue sans doúte par M. Simonin et présente comme poème liminaire aux Chroniques de Jean Carion philosophe 17 traduites par Le Blond, confirme sa position de curé de Branville - curé ayant une petite terre, ce qui l'autorisait à porter le titre de seigneur. Le terminus ad quem pour cette charge, donné par $\mathrm{M}$. Simonin, vient de ce poème : il aurait obtenu celle-ci en 1540, treize ans avant la publication des Chroniques de Jean Carion philosophe. Voici la pièce :

Le translateur dedie son livre à monsieur d'Evreux son treshonoré prelat, luy rendant compte de sa vissitation

Treize ans y a, c'est desja bien long terme,

(Dont, pour certain, devrois mourir de honte)

Que tiens de vous un petit lieu à ferme,

Sans vous avoir tenu compte.

Ce neantmoins que la somme ne monte

Pas à grand cos : noble prelat sacré :

Mais n'ay argent, parquoy ay consacré

En son endroit, à vous cestuy mien livre :

S'au lieu d'argent, papier vous vient à gré,

Je demourray, vers vous, quitte $\&$ delivre.

À part les distiques latins cités plus haut qui laissent entendre qu'il fut « instruit à Paris la docte», aucun texte ne fournit de renseignement sur son enfance.

Les dédicaces de ses ouvres permettent de connaître l'identité de ses protecteurs. Outre le prélat d'Évreux qui apparaît comme le dédicataire du poème précédent, on repère une dédicace à Claude d'Annebault, amiral et maréchal de France, en tête du premier volume du Livre de police humaine, la première œuvre traduite par Le Blond. ${ }^{18}$ Une autre se situe dans l'incipit d'une lettre introductive au Printemps de l'humble espérant, et renvoie au «baron de Ferieres seigneur de chambrays », auquel s'adresse son «treshumble serviteur ». Indépendamment des pièces de circonstances dont nous reparlerons, deux personnages bénéficient d'une attention particulière au début de deux ouvrages : le Dauphin de France (dans le Livre de police humaine) et le roi Henry II (dans Valère le Grand). Le Blond pouvait aussi compter sur ses amis et proches pour orner les premiers feuillets de ses œuvres. Parmi ceux-ci, deux sont récurrents : le lieutenant Mectayer (dans Valère le Grand ${ }^{19}$ et les Chroniques de Jean Carion) et Guillaume Le Saulnier (dans Le Printemps de l'humble espérant et les Chroniques de Jean Carion), pour qui Le Blond écrira un dizain en tête d'une de ses œuvres. ${ }^{20}$

17 Les Chroniques de Jean Carion philosophe. Trad. par Jean Le BLond. Paris, Jehan Ruelle, 1553

18 G. D'Aurigny, Le Livre de police humaine. Trad, par Jean Le Blond. Paris, Charles l'Angelié, 1544.

19 Valère le Grand en françoys. Trad, par Jean Le BLoND. Paris, Charles l'Angelier, 1548.

20 Dizain publié en tête de G. LE SAULNIER, Les décades de l'Esperant, qui est un sommaire et briefie interpretation de chascun chapitre des epistres de saint Paul (Rouen, 1550), signalé par M. Simonin, op. cit., p. 424. 
Ces quelques renseignements laissent apparaître Le Blond comme un homme de province religieux, cultivé et ayant reçu une éducation élevée qui lui ouvre les portes des cercles catholiques et royalistes. Il ne manque d'ailleurs jamais de saluer la famille royale au fil de ses œuvres.

Ces trois axes constituent le fondement de notre hypothèse concernant la trajectoire de Le Blond. Sa production sera ainsi à envisager selon trois points de vue : littéraire, avec les questions touchant à la défense et à l'illustration (poésie et traduction) par Le Blond de la langue française ; religieux, avec les troubles que connaît la chrétienté et son choix de parti ; et dans une moindre mesure, politique, avec l'allégeance constante de Le Blond au pouvoir royal français et ses traductions de recueils politiques de conseils au prince.

\section{Le Blond poète}

La production poétique de Le Blond est assez limitée dans le temps. Le premier manuscrit qui reprend un poème signé de son nom est le manuscrit français cote 24408 de la B.N.F. Il s'agit d'un recueil daté vraisemblablement de $1533,{ }^{21}$ qui comprend principalement des auteurs de 1520 à 1530 ayant participé au Puy de la Conception de Rouen. Le Blond y aurait pris part en 1533. Cette participation serait à replacer dans une étude plus vaste, ${ }^{22}$ analysant les rapports entre Marot père et fils, Sagon, Le Blond et d'autres ayant pris part à ces concours, avant la fameuse querelle. D'un point de vue littéraire, Marot et Sagon font en effet allusion, lors de la querelle, à leur participation à ce concours de palinods, Marot reprochant à Sagon d'être venu le trouver pour peaufiner une de ses œuvres, et Sagon accusant Marot de n'avoir jamais gagné. ${ }^{23}$

La poésie de ces concours est proche, par son style, de celle produite par les mal nommés Grands Rhétoriqueurs. Un décalage existe entre cette poésie et celle, contemporaine, de Paris. Outre le traditionnel retard périphérique de la province, le conservatisme idéologique des participants n'incite pas à la nouveauté.

Dans la même veine, le premier recueil de Le Blond s'inscrit dans la tradition poétique de la génération précédente : ce Printemps de l'humble espérant, daté de 1536, contient différentes pièces, ballades, blasons, rondeaux, oraisons, plaintes, dont la poétique est héritée directement de la fin du siècle précédent. $L e$ Temple de Diane et plaisir de la chasse, par exemple, renvoie très directement aux temples créés par Molinet, Lemaire de Belges ou encore Clément Marot dans sa jeunesse.

21 Voir M. Simonin, op. cit., p. 411.

22 Voir T. Mantovani, qui esquisse une telle étude. T. Mavrovani, «La querelle de Marot et de Sagon : essai de mise au point », dans La génération Marot [ ...], op. cit., pp. 381-404.

23 Voir C. MAROT, Le valet de Marot contre Sagon, cum commento (1557) et F. SAGon, Le rabais du caquet de Fripelippes et de Marot dict Rat pelé adictionné avec le commentaire, faict par Mathieu de Boutigny page de maistre François de Sagon secrétaire de l'abbé de Sainct Ebvroul (s.l.n.d.).
Ainsi, dans les commentaires de la querelle entre Sagon et Marot, les critiques n'ont pas manqué de souligner cette opposition. C. Kinch, en 1940, explique déjà :

Voici deux poétiques face à face. Sagon représente, pour ainsi dire, l'ancienne école savante et compliquée en présence de la poésie nouvelle, légère et courtisane, dont Marot est le chef incontesté 24

Le Blond, au vu de sa production littéraire de l'époque, est considéré sur le même pied que Sagon et La Huetterie, les deux plus virulents adversaires de Marot. Mais s'arrêter à cette constatation reviendrait à généraliser hâtivement. En effet, Le Printemps de l'humble espérant contient deux épîtres répondant à celle de Marot, Au Roy, du temps de son exil à Ferrare. Ces deux épîtres, dont il a été question plus haut, sont signées respectivement Jean Le Blond et le général Chambor. C.-A. Mayer a bien montré, vu les contradictions internes et les différences de style, qu'il ne peut s'agir du même auteur. Il importe de rappeler ici l'argument littéraire : les préférences et les références littéraires de chaque auteur sont très différentes.

Le Blond reconnaît les dons littéraires de Marot :

Le seigneur dieu, plain d'immortalité,

T'avoyt tant faict de liberalité

De ses haultz dons de grant parfection

C'est assavoir doulceur, invention,

Sy que tu eusse en fin peu adquerir

Loz \& triumphe \& amplement fleurir

Sur le throne d'eloquence françoyse.

Voyre au plus hault (\& pry qu'i[1] n'en desplaise

A toutz facteurs), car ta veine feconde

De beau parler n'avoyt point de seconde,

Mais ton delict \& vil Impurité

En ont perdu toute la dignité, [...] (vv, 143-154)

Pour Le Blond, Marot n'a pas d'égal. Il est le «Prince des poètes », ainsi que d'autres l'ont nommé. Le général Chambor, au contraire, condamne Marot sans appel :

De France dys, en ton oblicque escript

Que tu estoys son glorieulx poete,

Ou moins entendz que une chaulve chouette,

Qui ce qu'elle oyt essaye à gazouiller (vv. 180-183).

Le reproche est cinglant : Marot copie, et en plus le fait mal! Un profond désaccord oppose donc les deux auteurs d' «epistres responsives ». Il nous semble donc nécessaire de dissocier les trois attaques (Sagon, Le Blond et le généra Chambor) que Marot subit à la suite de sa lettre au roi. Dans un premier temps, il importe de distinguer l'attaque de Sagon de celles de la paire formée par Le Blond et Chambor. En effet, aucun de ces derniers ne prendra part à la querelle

24 Voir C. КіNCH, op. cit., p. 218. 
par la suite, échaudés peut-être, ou ayant épuisé leurs arguments et ne voulant pas tomber dans les grossièretés de circonstance. La prolongation de la dispute n'apportera rien de neuf au niveau du fond : bordées d'insultes succéderont à une collection de métaphores plus graveleuses les unes que les autres, si bien que même le public se lassera. On a souvent vu dans l'attaque de Sagon et dans celles de ses partisans de basses tentatives de positionnement à la cour. Si tel semble bien être le cas pour Sagon lui-même et pour La Huetterie, ainsi que pour leurs épigones ayant pris part à la querelle au fil d'incendiaires opuscules, cette opinion commune ne peut s'appliquer aux textes de Chambor et Le Blond. Le premier paraît un bien piètre poète pour prétendre rivaliser avec quiconque. Le second, on l'a vu, ne conteste pas la suprématie des dons de Marot. Il faut plutôt voir dans ces deux pamphlets la réaction épidermique de catholiques orthodoxes. L'un réfute en bloc l'homme et le poète, et l'autre découvre que Marot-poète qu'il admirait - est perméable à des idées qui le hérissent.

Deux écoles poétiques s'affrontent donc bel et bien, mais il ne s'agit pas du seul argument qui a poussé les belligérants à prendre part à la querelle. Dans le cas de Le Blond, c'est le ton trop « réformé » des derniers textes de Marot qui l'incite à prendre la plume. On verra à quel point la foi catholique est une préoccupation constante chez Le Blond. Mais, avant d'en venir à la religion, il faut s'attarder sur la position de Le Blond par rapport à la défense du français et à la traduction.

Le Blond, après la poésie, s'attache à la traduction du latin en français. La première qu'il donne est celle de la compilation latine extraite par Gilles d'Aurigny du grand traité de Francesco Patrizzi. Elle s'intitule: Le Livre de police humaine, contenant briefive description de plusieurs choses dignes de mémoire [...] nouvellement traduict de Latin en Francois par maistre Jehan le Blond, curé de Branville : \& dedié à hault \& puissant seigneur messire Claude d'Annebault Admiral \& Mareschal de France, \& lieutenant general au gouvernement du pays de Normandie, soulz monseigneur le Dauphin. Cette traduction a bénéficié de plusieurs rééditions, ${ }^{25}$ qui, à partir de la deuxième (comme l'affirme G. Charlier contrairement à R. E. Hallowell), ${ }^{26}$ contiennent une sorte de défense

25 Du vivant supposé de l'auteur: Paris, Charles L'Angelié, 1544 , in- $8^{\circ}$; Paris, Charles l'Angelié, 1546, in-16, deux volumes ; Paris, 1549, in-16, deux volumes ; Paris, Guillaume Thibout, 1554. Dans cette édition, le livre es dit « reveu et corrigé depuis les derniers impremez ». Aucune indication n'apparaît quant à l'identité du réviseur et correcteur.

${ }^{26}$ Les deux critiques ne s'accordent pas sur ce point : pour G. CHARLER (op. cit., p. 337), « ces pages apparaiss[ent] soudain au second volume de la deuxième édition d'un ouvrage déjà connu », R. E. HALLowELL (« Jean Le "This brief essay of 12 pre humaine, a book of excerpts choseared as Gilles d'Aurigny from cesco Patrizzi [... 1549 is given as the official date of publicetion of this third Fronch edition of thenist, FranIt is interesting to note that the tho previous editions of the Live of his third French edition of the work [...]. contain the translator's apologia for the Frens après consultation des édition de 1544,1546 t 1554 (1', de la langue française avant la lettre, située à l'ouverture du tome deux, sous le titre de «Preambule, touchant la noblesse, grace, et tres-ancienne dignite de la langue Françoise, qui peult estre une allumette à enflammer toutes personnes gentilles, à soy exercer audict languaige, \& en la doulce faconde, \& divine poesie d'iceluy ».

Ses vues sur la langue, extraites de ce petit essai, sont à mettre en perspective avec celles de La Deffence, et illustration de la langue françoise de Du Bellay. En effet, celui-ci a dû connaître le travail de Le Blond. C'est du moins l'hypothèse de G. Charlier :

Du Bellay a-t-il connu le Preambule de son devancier? Il est difficile de rien affirmer Toutefois divers indices rendent l'hypothèse assez séduisante. N'est-il pas curieux, par exemple, que l'on retrouve dans ces quelques pages plusieurs des citations ou des thèmes de la Deffence ? Les deux auteurs tirent gloire de la légende de l'Hercule gaulois : tous deux invoquent Cicéron et son Honos nutrit artes ; tous deux empruntent à Jean Le Maire le même passage sur le fabuleux Bardus V. Sans doute, chacun de ces traits, en soi, était banal. Mais qu'à trois reprises Du Bellay se soit rencontré avec Le Blond, c'est un hasard assez singulier s'il n'a point connu son opuscule, paru trois ans plus tôt et réimprimé au moment même où s'élaborait le manifeste de la Pléiade. 27

Pour venir étayer cette connaissance de Le Blond par Du Bellay, il faut s'attarder sur l'imprimeur de Le Blond. Les premières éditions des œuvres de Le Blond sont toutes publiées chez le même imprimeur: Charles L'Angelier, qui travaille avec son frère Arnould. ${ }^{28}$ Ceux-ci ont été fort peu étudiés : seul le mémoire de J. Renouard, conservé à la Réserve des imprimés de la B.N.F.,29 apporte quelques renseignements. J.-C. Monferran consacre un paragraphe à cet imprimeur :

Les trois premières éditions de la Deffence sont l'ouvre d'Arnould l'Angelier, libraire parisien qui, depuis 1536, tenait un étal dans la grande salle du Palais et qui publia, souvent avec son frère Charles, des ouvrages juridiques et médicaux, des traductions d'auteurs anciens et surtout des œuvres littéraires d'auteurs contemporains. ${ }^{30}$

En conclusion, il est fort matériellement possible que Du Bellay ait parcouru Le Printemps de l'humble espérant et lu le «Preambule» de Le Blond, vu 1'identité de leurs imprimeurs.

De plus, Du Bellay, dans le second livre de La Deffence, s'en prend à Le Blond par deux fois :

O combien je desire voir secher ces Printems, chatier ces Petites Jeunesses, rabattre ces Coups d'essay, tarir ces Fontaines, bref, abolir tous ces beaux tiltres assez suffisans pour degouter Lecteur sçavant d'en lire d'avantaige! Je ne souhaite moins, que ces

27 G. ChARLIER, op. cit., p. 342

28 Sur la couverture de la première édition (1544) du Livre de police humaine, il est précisé : «On les vend à Paris par Charles l'Angelié, tenant sa boutique au premier pillier du Palais, devant la chapelle de Messieurs les Presidens : \& en la rue de la vieille drapperie, pres saincte Croix, au logis dudict l'Angelié ».

29 J. Renouard, L'officine des L'Angelier, Réserve des imprimés, B.N.F., 1997.

30 J. Du Bellay, La Deffence, et illustration de la langue francoise. Éd. par Jean-Charles MonfERran. Genève, Droz, 2001, p. 47. TEXTES LITTERAIRES FRANCAIS, 543. 
Despourveuz, ces humbles Esperans, ces Banniz de lyesse, ces Esclaves, ces Traverseurs soient renvoyés à la Table ronde : et ces belles petites devises aux Gentilzhommes, et Damoyzelles, d'où on les a empruntées. ${ }^{31}$

Le Printems et l'humble Espérant sont donc condamnés, au même titre que le Depourveuz, que la critique identifie à l'Épître du dépourveu de Clément Marot. L'usage des devises l'est également. Deux esthétiques s'opposent : rappelons les compliments mêlés de reproches moraux que Le Blond adresse à Marot dans Le Printemps de l'humble espérant pour comprendre pourquoi ils sont ici réunis. Le contenu du «Preambule» ne fait que confirmer ce contraste entre les positions littéraires : là où, dans le chapitre III de La Deffence, Du Bellay attribue la pauvreté de la langue à «l'ignorance de notz majeurs », Le Blond s'en prend pour sa part à «l'injure du temps et negligence des hommes », et conclut par un appel à continuer le travail d'« illustration » de la langue déjà entamé :

Donnons ordre (nobles facteurs, poetes, \& orateurs François) chascun en son endroict, de la faire revivre, \& renaistre plus florissante qu'elle ne fut jamais, ainsi qu'on y a desja bien commencé par ouvres louables \& fructueux, \& conducibles, comme proses, oraisons liées, nobles poesies, $\&$ belles traductions. ${ }^{32}$

Contrairement à Du Bellay, qui chasse nommément ceux qu'il ne veut pas lire, Le Blond les exhorte à poursuivre, et il suffit de se souvenir de ses propres poèmes et des qualificatifs attribués à Marot par ses soins à propos de sa production de jeunesse pour comprendre quel type d'œuvres il souhaite voir prospérer.

Du Bellay, en reniant les poèmes de 1'ancienne génération, adopte une perspective historique moderne, avec une conscience du progrès propre à la langue. En s'opposant au passé proche, il pose ses propres choix comme seule possibilité. J. Balsamo l'exprime clairement dans Les Rencontres des Muses :

L'on sait avec quel mépris Du Bellay considérait les ouvres de l'ancienne école poétique, qu'il connaissait du reste très mal. La critique qu'il formulait à l'égard des prédécesseurs reposait sur l'écart qui séparait les genres poétiques qu'ils cultivaient et les modèles antiques : mais cette critique était indispensable parce qu'elle lui permettait de se démarquer de ce qui lui préexistait, pour mieux affirmer ses propres choix, dans une logique du tiers exclu toujours si forte dans la culture française. ${ }^{33}$

G. Charlier était du même avis en envisageant le cas de Le Blond : Du Bellay s'est opposé à lui, a fait siennes certaines idées, pour mieux briller. L'opposition ne joue pas uniquement dans les intentions, mais existe aussi dans les faits. Ainsi, l'usage du mythe de l'origine de la langue française sert un projet différent d'un point de vue esthétique selon les auteurs. Il en est de même pour la vision du poète inspirée de Cicéron.

31 lbid., pp. 168-169.

32 J. Le BLOND, « Petit preambule du translateur, touchant la noblesse, grace, \& tresancienne dignité de la langue Françoise ", dans G. D’Aurıany, Le Livre de police humaine. Trad. par Jean Le Blond. Paris, Charles l'Angelié, 1546, livre 2, f. 5 .

33 J. BALSAMO, Les Rencontres des Muses. Italianismes et anti-italianisme dans les lettres françaises de la fin du XVY siècle. Genève, Slatkine, 1992, BrblothèquU Franco Simone.
Cette opposition esthétique, première pomme de discorde, est complétée par une démarche différente concernant la traduction. Le fossé entre les générations, indépendamment de leurs choix littéraires, se marque dans la valeur et la légitimité accordées aux traductions.

Du Bellay voit le paradoxe qui existe à s'inspirer d'une autre langue pour affirmer les potentialités de la sienne et en afficher la grandeur. Le chapitre $V$ de La Deffence s'intitule «Que les Traductions ne sont pas suffisantes pour donner perfection à la langue Françoyse ». En effet, pour Du Bellay,

ce tant louable labeur de traduyre ne me semble moyen unique, et suffisant, pour elever nostre vulgaire à l'egal, et Parangon des autres plus fameuses Langues. [...] Je ne croyray jamais qu'on puisse bien apprendre tout cela [entre autres, les Methaphores, Alegoray jamais qu' on puisse bien apprendre tout cela [entre autres, les Methaphores, Alego-
ries, Comparaisons, Similitudes, Energies, et tant d'autres figures, et ornemens, sans les ries, Comparaisons, Similitudes, Energies, et tant dautres figures, et ornemens, sans les
quelz tout oraison, et Poëme sont nudz, manques, et debiles] des Traducteurs, pour ce qu'il est impossible de le rendre avecques la mesme grace, dont l'Autheur en a usé : d'autant que chacune Langue a je ne sçay quoy propre seulement à elle, dont si vous efforcez exprimer le Naif en une autre Langue observant la Loy de traduyre, qui est n'espacier point hors des Limites de l'Aucteur, vostre Diction sera contrainte, froide, et de mauvaise grace. ${ }^{34}$

Du Bellay n'est pas opposé à la traduction, au contraire. Mais il la juge insuffisante pour affirmer l'originalité et l'unicité du français. Le débat dépasse le simple enrichissement de la langue, pour s'élever jusqu'à l'essence même de cette langue française par rapport aux autres.

Le Blond, de son côté, n'envisage pas les termes du débat sous cet angle. «Translateur » estimé, du moins de ses amis, ${ }^{35}$ il se contente de quelques mises en garde sur l'usage que l'on peut faire des traductions.

Et si aucuns veulent dire, que par traductions, \& languaiges tournez de Latin en terme François, est advenu du mal, je responds que ce n'est la faulte de nostre langue, mais le vice des abusans. L'or est ministre de bien \& de mal, l'argent, les chevaulx de quoy on faict la guerre Origene dict que les choses qui sont bonnes de nature, quand nous abusons d'icelles, nous menent à péché. La langue Françoise peult estre ministre de grand vanité, en couchant en icelle choses lascives \& scandaleuses : mais comme j'ay dict au commencement, il n'est rien pire qu'une mauvaise langue. ${ }^{36}$

Le Blond se contente donc d'objections d'ordre moral. Il n'est nullement question de spécificités propres à la langue, ni d'allusions linguistiques. Les seules observations qu'il fera sur la traduction sont d'ordre pratique, et sont contenues dans une brève note annexée à la traduction de l'Utopie. Nous y reviendrons plus loin.

Ces remarques morales à propos de la traduction soulignent l'importance que Le Blond accorde à la morale catholique. Cette facette de sa vie est à prendre en compte pour comprendre une des raisons qui le poussent à écrire puis à traduire.

\footnotetext{
34 J. DU BELLAY, op. cit, pp. 85-88.

35 Plusieurs poèmes en tête de ses différents volumes de traduction le louent grandement.
}

36 Jean Le BLoND, « Petit preambule du translateur [...]», op, cit., f. 4. 


\section{Le Blond catholique}

Jean Le Blond était curé de Branville (cure seigneuriale), comme le précise le titre du Livre de police humaine. Plus tard, il dédiera les Chroniques de Jean Carion à M. d'Évreux, son «trèshonoré prelat». Cette fonction ecclésiastique est loin d'être un pis-aller : son investissement dans la foi catholique est présent dans toute son ouvre.

Son premier poème connu est celui qui lui permit de prendre part au concours des palinods de Rouen, lors duquel, pour M. Simonin,

il s'agit au reste bien moins pour les élites d'affirmer un quelconque talent poétique que de manifester leur solidarité et, dès les années 1530 , au travers du culte marial, leur attachement à la religion traditionnelle. ${ }^{37}$

Le contenu de son premier recueil - Le Printemps de l'humble espérant regorge de références à la religion catholique et au culte marial : les titres des poèmes sont, de ce point de vue, éloquents : «Epistre a la fyebvre quarte qui avoyt prins ung Evescque », « Plainte sur le trespas de maistre benoist de la noe docteur en theologie penitentier Devreux », «Vers septains a la louenge de limmaculee Vierge Marie », «Aultre rythme en lhonneur de linviolee virge \& treschaste marie », «Dizain a ung orfebvre expugnateur des lutheriens », « Dung cœur contri presente a nostre saulveur Jesuscrist », «Vers alexandrins contre les profanateurs de sainct lieu », «Plaincte sur le trespas de tresdevote \& treschaste dame Jehanne Darneiuville en son vivant abesse de sainct sauveur devreux ou sont introdiuctes troys dames virginite, humilite \& raison », « Oraison tresdevote a limmaculee $\&$ tressacree virge mere de dieu marie pour Impetrer a bien vivre \& bien mourir ", «Ballade dung may plante devant leglise nostre Dame de Paris en lhonneur de la tressacree Vierge », «Rondeau a la vierge marie ou par les lettres capitales on trouvera Marie Vierge ». Environ la moitié des poèmes du recueil ont ainsi un rapport étroit avec la religion catholique.

L'«Epistre à Jean Chrestien, envoyé aux chants Elysées par le vent Zéphire », est à rapprocher des pièces religieuses ; concernant celle-ci, l'abbé Goujet ajoute :

[lettre] dans laquelle il déplore la mort de cet ami qui avoit été tué dans quelques émeutes des Religionaires. 38

On pourrait peut-être voir dans ce personnage de Jean Chrestien une personnification des catholiques qui, d'après Le Blond, devaient endurer de fortes pressions luthériennes. M. Simonin parle « d'une Normandie autrement touchée par la Réforme que ne l'est le Paris de ses études [celles de Le Blond] ».39

\footnotetext{
37 M. Simonin, op. cit., p. 234.

38 Abbé GouJet, op. cit., p. 109

39 M. Simonin, op, cit, p. 409
}

En conséquence, cette forte préoccupation religieuse se confond souvent avec un antiluthérianisme certain. Ainsi, dans le «Dizain à ung orfebvre expugnateur des lutheriens » :

Vray zelateur de la foy treschretienne

Contre l'erreur de luther reprouvé,

Ferme pilier, fort et constant Estienne ;

Nostre bon dieu en ce point t'a prouvé

Ainsi que l'or au feu est esprouvé,

Tu fuz trouvé, guerny de purite,

Tousjours loyal soustenant verité,

A ton pouvoir pour garder d'interest

La saincte loy en son integrité ;

Au grant besoing voyt on qui amy est. 40

C.-A. Mayer, dans une note qui commente ce dizain, précise :

sans doute la Réforme avait-elle pris une ampleur en Normandie qui effrayait les croyants orthodoxes. Ce n'est peut-être pas une simple coïncidence que les ennemis les plus acharnés du poète [Clément Marot], Sagon et Le Blond, aient été normands.

En effet, pour Le Blond, parmi ceux qui ne veulent «garder d'interest la saincte loy en son integrité » s'est perdu Marot. L' « Epistre à Clement Marot responsive de celle parquoy il se pensoyt purger d'heresie lutheriane », publiée dans le même recueil, est assez explicite à ce sujet. Comme nous l'avons vu, Le Blond admire les dons littéraires de Marot, mais lui reproche le ton trop « réformé » de ses derniers textes. Il 1'exhorte à abandonner ces «propos mescheantz de tout l'estat monasticques en [s]es chantz » (vv. 29-30).

Ainsi, l'entrée en matière de l'épître n'adopte pas la prudence et le cérémonial des circonlocutions de Sagon : Le Blond reproche directement à Marot sa demande de retour au Roi :

Veux-tu que France, O deloyal ministre,

De treschrestienne en vain tienne le tiltre,

Et que celle qui tousjours fust nourrice

De vrays chrestiens monstres infaictz nourrisse?

Tant qu'il sera dans cette erreur, rien ne pourra être fait pour lui :

Estimes tu que celuy qui vouldroict

(Là où le cas semblable au tien viendroyt)

Ses vrays enfanz tainctz de tel malefice

Livrer luy mesme à en fayre justice,

Pour son honneur denigrer \& ternir,

En cest erreur te voulsist soustenir ? (vv. 13-22)

Les preuves de son double discours, de son penchant pour la Réforme, dorment de façon irréfutable dans ses livres et écrits. Pleuvent en effet dans ceux-ci

40 Cité par C.-A. MAYER, La religion de Clément Marot, op. cit., p. 78, note 22. Contenu dans Jean LE BLoND, Le Printemps de l'humble espérant, op. cit. 
les reproches contre l'Église, les remises en question du dogme et les hérésies condamnables aux yeux de Le Blond.

Ce dernier entreprend la défense de l'Église sur un point précis - il cautionne l'enrichissement des hommes de foi par la première épître aux Corinthiens (vv. 41-46) - et revient ensuite à des propos plus larges concernant une excessive généralisation opérée selon lui par Marot : l'Église ne doit pas être condamnée dans sa globalité pour quelques fruits pourris qui s'y seraient glissés. Poursuivant son travail de justification point à point, Le Blond embraye sur la justice (vv. 80-94) : il ne veut pas croire Marot et sa description de l'Enfer, ${ }^{41}$ i réfute « le tort et l'injustice » des « suppostz de justice ». Il lui reproche de ne pas être objectif, et de vouloir prendre une revanche sur l'appareil judiciaire qui 1'a, selon Le Blond, justement condamné.

Sa défense de la Sorbonne est aveuglée par sa foi en la toute-puissance de son camp ; il prétend, à tort et contrairement au général Chambor, ${ }^{42}$ qu'il s'y enseigne les trois langues, le latin, le grec et l'hébreu :

Quant à ce point où ta plume s'advance

De reciter en dol \& decepvance

Que la Sorbonne ayt le grec deffendu ;

Car en leçons, en sermons ou harengues

On voyt fleurir les docteurs aux troys langues (vv, 116-120)

Pour justifier l'interdiction, Le Blond utilise et adapte au cas particulier de Marot un argument présent dans le texte de Chambor : la mauvaise interprétation que l'on peut donner aux textes sacrés en voulant les lire par soi-même.

Puys fol delict, où gist ton fondement,

Devant les yeux de ton entendement

Tend son rideau d'hombrageuse taincture

Dont tu sens mal de la saincte escripture,

Et la traduictz en ton sens depravé,

Ainsi qu'on a en tes escriptz trouvé (vv. 131-136).

Appuyé par plusieurs métaphores (une technique que Le Blond affectionne particulièrement), le reproche d'arrogance fuse ; traité de «présumptueux veau », Marot se fie trop à son sens critique :

\footnotetext{
41 On pourrait d'ailleurs voir daus ces vers une allusion directe à l'Enfer, bien qu'il ne fut publié par Dolet qu'en $1542 \ldots$ Peut-être un manuscrit pourrait-il avoir circulé auparavant :

Tu accuses les suppostz de justice,

Disant qu'à maintz font tort \& injustice,

Et, pour vouloir en tes dictz triumpher,

Tu te penses saulver en leur enfer,

Du quel tant bien sçais l'entrée \& l'yssue,

Et dont le front encore de peur te sue (vv. 81-86)

42 Mayer l'a déjà souligné: ce type d'incohérence montre à quel point les trois attaques (Sagon, Le Blond, Chambor) contre Marot étaient peu concertées. Voir C.-A. MAYER, «Clément Marot et le Général de Caen », op.
}

Ce neantmoins que tu ays abusé

De ton vray sens \& long temps mal usé,

Sy penses tu estre plus entendu

Que tout le monde, \& tout seul descendu

De Juppiter, créé en son cerveau (vv. 163-167).

La liberté du poète, revendiquée par Marot dans son épître $A u$ Roy, $d u$ temps de son exil à Ferrare, est limitée explicitement par Le Blond : elle ne peut aller contre la loi chrétienne (vv. 179-182). Ce corsetage de la science et de l'esprit critique est justifié par Dieu :

Car l'inventeur ne veult pas qu'on abuze

De la science en l'esperit infuse, [...] (vv. 183-184).

Le Blond arrive alors au problème qui l'irrite le plus, en catholique orthodoxe qu'il est : Marot a osé s'élever contre les bûchers d'hérétiques.

Tu dys que c'est grosse confusion

Tes compaignons \& alliez amys

Lutheriens, [...] (vv. 188-191).

C'est l'occasion pour Le Blond de déverser tout son fiel contre cette « secte plaine d'envye » (v. 191). Ces vers préfigurent ceux qui viendront dans la suite de la querelle sous d'autres plumes : insultes, emportements et envolées intégristes contre les hérétiques. Dans la mesure où Marot plaint ces gens, il mérite, au même titre qu'eux, le bûcher.

Le Blond apparaît ainsi comme un chrétien orthodoxe, admiratif de Marot par le passé. Il supporte très mal les erreurs présentes de ce dernier, et le lui signifie de manière violente afin de le ramener dans le droit chemin.

À la lecture de ces mots, on peut s'étonner de l'intolérance qui habite le futur traducteur de l'Utopie. Quatorze ans séparent ces textes, et beaucoup d'événements ont amené Le Blond à prendre plus de recul par rapport aux mesures de gouvernance politique. Sa production littéraire va d'ailleurs, après ce recueil, s'orienter vers des traductions de traités politiques et de conseils aux princes, puis des chroniques historiques.

\section{Le Blond conseiller du prince}

La deuxième auvre de Le Blond, que l'on situe en 1536, juste avant le recueil Le Printemps de l'humble espérant, s'intitule La deploration sur le trespas de feu monseigneur le Dauphin de France. Avec l'epitaphe dudict seigneur. et ung dizain a la louange du Roy pour sa constance contre Fortune et Mort. Ensemble l'epitaphe du comte Dampmartin. Un an plus tard paraissent à Paris également les Nuptiaul $x^{43}$ virelays du mariage du roy d'Escoce et de madame

43 Et non Nowreaux [...], comme l'écrit M. Simonin dans l'article consacré à Le Blond dans le Dictionnaire des lettres françaises (M SMovin «Jean Le Blond", dans Dictionnaire des lettres francuives, Le seizième siècle.

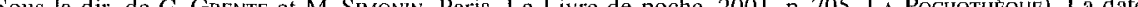


Magdaleine, première fille de France, ensemble une ballade de l'apparition des troys déesses, avec le blazon de la cosse en laquelle a tousjours germiné la belle fleur de lys, faict par Branville. Il s'agit de la dernière pièce isolée publiée par Le Blond. Celle-ci, lancée au milieu d'une profusion d'autres célébrant le même événement, ainsi que la première, plus isolée, soulignent l'intérêt que présente déjà Le Blond pour la chose politique et les puissants. Sa voix, pour le second poème, semble noyée pour la postérité parmi les gloires présentes (Marot) ou futures (Ronsard), ou même les anonymes (un poète dont la devise est « vouloir selon le pouvoir $\gg$ ), qui commémorent ce mariage. M. Simonin en cite plusieurs, et souligne la particularité de Le Blond:

De tous, c'est Le Blond qui adopte le ton le plus politique ; pour lui, il s'agit bien de célébrer une union avec "Ung peuple amy, loyal et secourable, / Aux fleurs de lys de tout temps favorable $» .44$

À la suite de ce poème, la principale activité de Le Blond, curé, avocat et poète, durant les seize années suivantes, sera le conseil aux puissants par l'intermédiaire de traductions.

C'est dans cette catégorie que l'on peut classer le Livre de police humaine, sa première traduction, dont il a déjà été question. Dès la deuxième édition du livre (1546), le texte d'Érasme destiné à « l'enseignement du prince chrestien » est joint aux extraits de Francesco Patrizzi «contenant briefve description de plusieurs choses dignes de mémoire : sicomme du gouvernement d'ung Royaume, $\&$ de toute administration de la Republique $\gg .45$

Dans sa dédicace à Claude d'Annebault, Le Blond explique qu'il pense servir le pouvoir et faire œuvre utile au moyen des conseils prodigués dans ce livre

Parquoy estimant grandement ma bienheureté, d'estre du nombre de vos hommes, \& tresobeyssans subjectz (voire le moindre en sçavoir et vertu) natif en vostre territoire d'Evreux : en tesmoignage de gratitude, jay entreprins de tourner en nostre vulgaire François \& à vous dedier ledict petit recueil qui a esté de bien nouveau, extraict des amples \& grandz volumes de Françoys Patrice, natif de Sennes en Italie, auquel est parlé souverainement du gouvernement et regime de la chose publique. Et pour autant qu'ung bon pilote de mer (ainsi comme le recite le treselegant poete Ovide) prend grand plaisi à deviser \& à ouyr parler des ventz, \& choses qui concernent le faict de la mer, un laboureur de ses beufz \& de sa charue. Par semblable m'est il advis qu'un gouverneur du bien public, \& amyable zelateur d'une police humaine, est grandement joyeux, quand il oyt tenir propos conducibles \& fructueux de son estat. 46

mort de Le Blond dans ce dictionnaire est aussi erronée : 1553 est annoncée, alors que la dédicace de sa dernière cuvre, L'histoire de Normandie, est adressée à « Monseigneur Monsieur Rober[t] de Quenel, abbé de la Noe et de Conches» (fol. II $v^{\circ}$ ). Or, G. Charlier explique que « " c'est seulement en 1558 que Robert de Quesnel dut à 1 aveur de Diane de Poitiers ces deux abbayes normandes " (G. ChaRLIER, op. cit., p. 336). Le manuscrit étant u Le Blond, il s'agit là de la dernière date assurée dans la biographie de l'auteur.

44 M. SimonNN, « À chacun son fréron [...] », op. cit., pp. 414-415.

45 Page de titre du Livre de police humaine, édition de 1546 ( op. cit.).

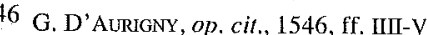

Le Blond considère donc le résultat de son travail comme un délassement en rapport avec la gestion du bien public, un ouvrage dèstiné à divertir plaisamment tout en instruisant. Il présente sa démarche comme découlant de l'exemple d'une autorité reconnue, Ovide. Cette manière de procéder revient régulièrement dans ses écrits ; il avance rarement un jugement sans le justifier. Ce recours constant à ses lectures constitue un excellent indicateur de son niveau de culture et de ses connaissances directes ou indirectes.

La conclusion de sa dédicace exprime très clairement les objectifs de son projet de traduction, et éveille un intérêt particulier concernant le but de la traduction de l'Utopie:

quant à moy, j'ay c'este ferme confiance, que daignerez estendre les rayons luysans de vostre gratieux æil, sur ce livret que j'ay intitulé, Le livre de Police humaine, pource qu'en ensuyvant ce qui est comprins en iceluy, l'homme vivra en ce monde glorieusement en honneur et bruyt, \& sera guidé à la fin desirée \& heureuse. 47

À partir de ce moment, il n'abandonnera jamais la tâche qu'il s'est assignée : par ses écrits, il entend mener l'homme vers une vie glorieuse et une fin tant désirée qu'heureuse.

La deuxième œuvre de didactisme politique qu'il traduit est un abrégé de Valère Maxime: Valère le Grand en Françoys, où sont comprins les faictz et dictz dignes de memoire, tant des vertueux personnaiges que des vitieux, affin que les hommes par la splendeur \& beaulté des vertuz, soient enflammez à les ensuivir : pareillement par la turpitude \& reproche des vices, soient incitez à fuir \& avoir horreur d'iceux [...] dedié au Treschrestien Roy Henry, deuxiesme de ce nom, Paris, Charles l'Angelier, 1548. Le titre, explicite à souhait, situe le contenu du côté de l'exemplum. De plus, Le Blond, dans l'abrégé de la vie de Valère Maxime qu'il rédige en guise d'introduction, présente cette traduction comme répondant à un objectif pédagogique :

Quelque temps fut soudard, \& feit le navigage d'Asie, avec Sextus Pompeius. Luy revenu, voyant qu'il pouvait apporter fruict à son pays, tant en bien disant, qu'en bien faisant, retourna à l'estude, dont il avoit esté destourné, par le desir de veoir la guerre \& delibera d'escrire les faictz \& dictz dignes de memoire des Rommains, \& nations estranges, ainsi comme luymesme le confesse. ${ }^{48}$

«Apporter fruict à son pays » apparaît comme la revendication principale de Le Blond. Dans la «Dedication de ce present livre, au roy treschrestien Henry, deuxiesme de ce nom », on comprend que les conseils contenus dans le livre s'adressent maintenant au peuple français, successeur des Romains si l'on suit le raisonnement de Le Blond :

Ne prens plus gloire en moy, plus ne suis tien

Romme qui fut la princesse du monde.

France m'a fait par un escripvain sien,

Puis peu de jours tourner en sa faconde.

47 G. D'AURIGNY, op. cit., 1546, f. VI.

48 Valère le Grand en francoys. Trad de Jean Le BLovd, Paris, Charles l'Angelier, 1548 , f V. 
Si ton Tybere en murmure ou en gronde,

Il ne m'en chault : Françoys ore me nomme.

Et si tu quiers de quel sieur me renomme,

La ville aussi, ou je me veulx tenir :

Je te respons que Paris cest ma Romme

Et mon Caesar, Henry, pour l'advenir.

La traduction de l'Utopie, publiée en 1550, s'inscrit directement dans cette lignée d'utilité publique. Les deux derniers ouvrages connus de Le Blond sont des chroniques : Les Chroniques de Jean Carion philosophe se complètent des faits et gestes de François ${ }^{\text {er }}$ et de Henri II, rédigés par Le Blond lui-même, tandis que La Chronique de Neustrie est uniquement de la plume de Le Blond. Celui-ci définit, dans une introduction à sa chronique de François $\mathrm{I}^{\mathrm{er}}$, les objectifs de son travail. Après avoir différencié le chroniqueur de 1'historien, il se donne comme chroniqueur et explique :

Ce que j'ay faict en ceste presente Chronique. J'ay assemblé seulement (comme en un abregé) les choses qui m'ont semblé plus necessaires, \& aucunesfois en passant enseigné les occasions d'icelles, afin que plus diligemment un chascun aprenne à se mirer aux faicts d'autruy, \& que par les yssues \& adventures d'iceulx, se delibere d'ensuyvir dont peut advenir honneur, \& fuyr la chose dont peut proceder reproche et infamie. Quant au reste, nous avons declaré au commencement, combien d'autres utilitez on reçoit en lisant une chroniques 49

Faire profiter autrui de 1'expérience acquise par l'histoire reste la véritable obsession de Le Blond. Pourtant, en ces temps troublés, il a perdu la confiance en l'avenir qui l'habitait lors de ses premières traductions. La suite du texte explique que la fin du monde est proche, et que la longévité prédite par le prophète Élie ne doit pas faire espérer : la mauvaise vie du monde va entraîner une révision des calculs divins. Et Le Blond de justifier son affirmation par une analyse qui fait intervenir ses deux domaines de prédilection, la religion et la politique des états. La déchéance des grands royaumes et empires et les troubles religieux de l'époque viennent le conforter dans son idée. Il évoque un second prophète, Daniel, et s'aperçoit que leurs prophéties concordent: d'après les tables chronologiques contenues dans la chronique de Carion, on arrive à la fin des six mille ans prévus par Élie, et la chute de l'empire turc est imminente, comme le prévoit Daniel. Même le ciel menace : les phénomènes célestes inquiétants se multiplient.

D'avantage, puis que nostre seigneur Jesus Christ en son evangile nous admonneste des

perilz qui viendront en la fin du monde, \& non seulement quant aux choses qui appar-

tiennent au corps : mais celles qui touchent l'ame : mesmes aussi le ciel nous menace

par ses horribles ecclipses \& conjunctions. 50

49 Les Chroniques de Jean Carion philosophe, op. cit., f. 263r". Bien que M. SImonin, dans son article «À chacun son frérion [...]» (op. cit.), renseigne une édition en 1550, nous citons d'après celle de 1553, car chah'avons pas pu consulter Jean CARION, Chroniques. Paris, E. Groulleau, 1550

50 Les Chroniques de Jean Carion philosophe, op, cit, f. $264 \mathrm{r}^{\prime \prime}$ et $\mathrm{v}^{\circ}$. Deux ćlipses de soleil eure Paris en moins de quatre ans: l'une le 12 novenbre 1547, l'autre le 31 août 1551 . Been que partielles, sur durent marquer les esprits par le faible intervalle qui les séparait. D'après Dominique Sluse, chercheur à l'Institut
Après cette chronique personnelle, introduite dans celle de Carion, il écrit La fondation du royaume de Neustrie maintenant Normandie, en la Gaulle Celtique par les François Allemans. Ce fort volume d'environ 680 feuillets doit encore être analysé. À première vue, il s'agit du même type de chroniques que celles à propos de François I ${ }^{\mathrm{er}}$ et Henri II accompagnant la chronique de Carion, à savoir une énumération d'événements extrêmement factuels, sans jugement ni explication.

La trajectoire sociale de Jean Le Blond est maintenant mieux connue, et ses principaux centres d'intérêt sont assez évidents. Comment la traduction de l'Utopie s'est-elle inscrite dans ce parcours? Quel était l'objectif poursuivi par Le Blond en traduisant cette grande œuvre humaniste? Quelle fut la lecture qu'il en eut? Nous proposons d'esquisser une réponse à la lumière des éléments de la première partie.

\section{La traduction de l'Utopie}

Notre propos s'articulera autour des trois mêmes axes, à savoir la langue, la religion et la politique.

Le volume paru en $1550 \mathrm{chez}$ Charles 1'Angelier, et intitulé La description de l'isle d'Utopie, où est comprins le miroer des republicques du monde, \& l'exemplaire de vie heureuse, se présente comme un in-octavo de 105 folios et VIII ex-folios. Il comporte, comme document accompagnant l'Utopie, la lettre de Guillaume Budé à Thomas Lupset, publiée en 1517 dans l'édition de Gilles de Gourmont, et traduite également par Le Blond.

\section{La langue de l'Utopie}

On l'a vu, Le Blond a une haute idée de la pratique traductive. Il y voit un moyen pour le français de s'enrichir et de s'imposer comme « le choix, le triumphe, \& l'eslite du monde ».51 L'Utopie lui a posé certains problèmes, qu'il exprime dans un avertissement situé en fin de volume, intitulé «L'Autheur au lecteur » :

Ne sois offence amy lecteur, si en ceste mesme petite tradiction, tu trouves oultre les loix \& reigles de tourner quelque oeuvre, que j'aye aulcunefois usé de Paraphrases. Je lay faict pour rendre les sentences de lautheur plus intelligibles. Et consequemment si en

d'astrophysique et de géophysique de l'Université de Liège, celle de 1547 devait être la plus impressionnante des d'astrophysique et de géophysique de ${ }^{\prime}$ Université de Liege, celle de 1547 devait être la plus impressionnante des
deux, car, si elle était partielle, elle n'était pas annulaire : elle a donc caché une plus grande partie du soleil. II de $95 \%$ proportione que ces deux éclipses étaient loin d' atteindre. On qui signifierait, s'il n'est pas empetique que oe prêche fit rédigć spécicont pour l'ćdition de 1553 (et

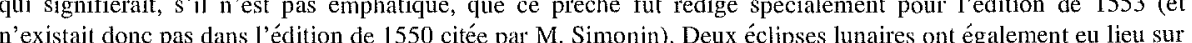

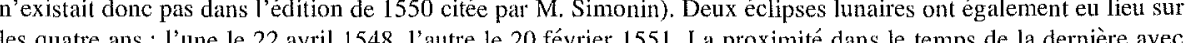

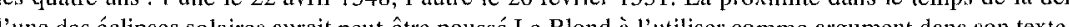
51 Dans G. D'AurignY, op. cii., 1544, f. VII. 
traduisant j'ay ramené en nostre usaige françois certains termes infrequentz. On ne se doibt mal contenter si un personnaige faict renaistre \& reduit en cours quelques vocables trouvez en autheurs Idoines, \& sil sefforce donner nouveaulté aux parolles anciennes, \& ne souffre totalement perir les motz qui par la coulpe des temps sont tournez en desacoustumance. En sorte que si nous n'usions que de termes vulgaires \& communz à chascun, nostre langue nen enrichiroit d'un flocquet, \& fauldroit tousjours faire comme les tabellions \& notaires, qui en leurs actes ne changent ne ne muent de stille. 52

Les paraphrases et les mots hors d'usage ont suffisamment tracassé le traducteur pour qu'il prenne la peine de s'en excuser auprès du lecteur. Il faut dire que la langue de l'Utopie, variée et ludique, a suscité des polémiques jusqu'à nos jours. On peut citer par exemple le compte rendu par A. Prévost de la réimpression de la traduction de M. Delcourt :53 il lui reproche la mauvaise interprétation de nombreux passages, qu'il s'agisse des noms propres (Abraxa, Caliquit) ou de noms communs relevant d'un vocabulaire spécifique (religieux surtout).

Les noms propres ne semblent pas avoir posé de problèmes particuliers à Le Blond. Que certains aient des racines grecques ne paraît pas l'avoir perturbé, car il les traduit sans aucune remarque. Il en va de même pour le passage en grec de la lettre de Budé. Certains noms subissent une adaptation, voire une paraphrase, mais aucune remarque spécifique ne vient souligner la difficulté particulière qu'ils constituaient. Barthélemy Aneau, lors de la réimpression en 1559 de la traduction de Le Blond, adjoindra à celle-ci une «Interpretation sur les noms propres des personnes, choses ou circonstances, qui par l'autheur ont esté inventez \& formez à plaisir, \& à propos de l'histoire Utopique $\gg .54$

Au niveau du style et de la fidélité de la traduction de Le Blond par rapport à l'original, les avis modernes ne tarissent pas d'éloges. M. Jeanneret, dans son introduction à la réimpression de la traduction de Le Blond dans la collection dirigée par M. Screech, « Les Classiques de la Renaissance en France », conclut par ces mots :

Le Blond s'impose surtout comme traducteur ; la version de l'Utopie suffit à convaincre de son savoir-faire : il est à la fois fidèle et élégant et il faut souhaiter que la présente réimpression suscite une étude qui rende justice à la qualité de son travail..$^{55}$

R. E. Peggram, lors d'une comparaison entre la première traduction anglaise et la première traduction française de l'Utopie, écrit de même :

It is a work of ultra-refinement, designed to suit the precious tastes of the cultured nobility. [...] It was Le Blond who apologized for any possible vulgarisms in his works, but he was far too elegant to write any, ${ }^{56}$

52 Dans Th. More, La description de l'isle d'Utopie, op. cit., 1550, non paginé

53 A. Prévost, «Une rétrospective : le facsimilé de l'Utopie éditée par Marie Delcourt ", dans Moreana, $\mathrm{n}^{\mathrm{n}} 85$, 1985, pp. 67-82.

54 Th. More, La republique d'Utopie. Préface de Barthélemy AnEAu. Trad, de Jean Le Blond. Lyon, Jean Saugrain, 1559.

55 M. JEANNERET, «Introduction », dans Th. MoRE, La description de l'isle d'Utopie. Trad. de Jean Le BLOND. La Haye, Mouton, 1970, t. 2, pp. XIV-XV.

56 R. E. PeGGRAm, "The First French and English Translations of Sir Thomas More's Utopia», dans The Modern Language Review, t. 35,1940 , p. 337.
B. Hosington, comparant cinq traductions de l'Utopie, couronne son raisonnement en ces mots :

Le Blond's and Aneau's versions exhibit several characteristics of Renaissance translations, although their high level of accuracy is surprising : the use of two words for one, the occasional indulgence in a brief, added explanatory phrase, and a disregard for the overall tone and style of the original - their French is more elevated than More's overall tone 57

Même A. Prévost porte un jugement positif sur la traduction de Le Blond :

Cette version dans une langue élégante et proche du latin est remarquablement fídèle. Son exactitude et son style ont fait d'elle la traduction classique qui s'est imposée au public de langue française pendant plus d'un siècle. 58

«Langue proche du latin » ne doit pas s'entendre du point de vue tonal ou stylistique, c'est-à-dire comme la correspondance entre les moyens d'expression latins et leurs équivalents en français, mais plutôt comme le décalque du latin opéré par Le Blond dans les constructions de phrases, les groupements syntaxiques et autres tournures lexicales.

Malgré les précautions prises à propos de son travail de traducteur qui outrepasserait « les loix \& reigles de tourner quelque œuvre», Le Blond, d'un avis général, a élaboré une traduction raffinée d'un ouvrage délicat à traduire. Proche des principes de traduction défendus par Étienne Dolet, 59 il met le lecteur en garde et se justifie lorsqu'il s'en éloigne, comme lorsqu'il introduit des mots hors d'usage. La qualité de son travail et sa conscience professionnelle se confirment avec l'exemple de l'Utopie.

\section{La religion dans l'Utopie}

Le rapport à la religion de l'Utopie est fort controversé : les interprétations que l'on en a données vont d'un extrême à l'autre, certains en faisant un ouvrage marxiste avant la lettre, d'autres une sorte d'organon, qui fait de l'Utopie un instrument de «maïeutique de soi ». On peut trouver un bilan de beaucoup de ces interprétations dans l'ouvrage de C. Quarta, Tommaso Moro. Una reinterpretazione dell'«Utopia ».60

La foi de More est bien connue - nous n'en parlerons pas - , ce qui ne l'a pas empêché d'introduire dans son texte certaines critiques de la religion. Les gloses marginales constituent les remarques les plus acerbes de ce point de vue.

57 B. Hosington, op. cit., pp. 133-134

58 A. PRévost, «Introduction », dans L'Utopie de Thomas More. Présentation, Texte original, Apparat critique, Exégèse, Traduction et Notes. Éd. par A. Prévost. Paris, Mame, 1978.

59 Voir les cinq principes dans Étienne Dolet, La maniere de bien traduire d'une langue en aulire. D'avantage. De la punctuation de la langue Francoyse. Plus. Des accents d'ycelle. Lyon, chez Dolet mesme, MDXL. Réimpression : Genève, Slatkine Reprints, 1972.

60 C. QUARTA, Tomnaso Moro. Una reinterpretazione dell'" Utopia ». Bari, Dedalo, 1991. Nuova BıвLIOTECA Dedalo. 
Sur tout le chapitre sur la religion, dans l'édition de Gilles de Gourmont, on en dénombre quatorze (quinze dans l'édition bâloise de novembre). Le Blond n'en traduit que quatre, ${ }^{61}$ et évite les plus critiques. Si l'on en croit Hosington, Le Blond omet une glose sur trois dans le reste de l'ouvrage. La proportion est ici plus importante, ce qui ne doit pas vraiment étonner. Sa foi a pu le retenir de traduire les passages les plus critiques envers la religion, par exemple « at apud nos quanta turba est » ou « at apud nos qui sunt inquinatissimi aris proximi esse contendunt $»$.

En revanche, les coutumes religieuses des Utopiens ne font pas sourciller Le Blond. Si l'on s'intéresse à la façon dont il traduit les passages sur la religion à l'intérieur même du texte, on s'aperçoit qu'il apporte à ces extraits le même soin scrupuleux qu'au reste de l'ouvrage, avec une présence abondante de paraphrases, comme son avertissement le laissait pressentir, et l'utilisation d'un procédé rhétorique fort courant chez les traducteurs de la Renaissance : l'amplification. Seules les critiques directes envers la religion chrétienne semblent donc l'indisposer, étant donné qu'il ne les traduit pas. L'exposé d'autres coutumes religieuses et d'autres mœurs est traité de manière totalement neutre.

\section{L'Utopie, ou la meilleure forme de gouvernement}

Ouvrage politique sans aucun doute, l'Utopie s'inscrit dans les traductions d'œuvres politiques de Le Blond. Celui-ci a conscience du profit que tout gouvernant pourrait tirer de cette lecture, mais, comme souvent, il n'ose l'affirmer sans recourir à d'autres autorités. C'est pourquoi, dans une courte préface au lecteur, il justifie son choix en recourant à l'avis de Guillaume Budé, dont il reproduit la lettre jointe à la deuxième édition de l'Utopie.

Afin que tu ne penses Ami que de mien privé \& seul jugement je t'ay mis en lumière en nostre langue ceste description de l'Isle d'Utopie considerant comme il est escript que l'homme ne se doibt appuier sur son privé sens \& prudence \& aussi que au tesmoignage de deux ou de trois toute chose doibt estre aresstée non content du seul tesmoignage de Thomas Morus qui premier a redigé en latin ladicte description je me suis grandement fondé sur ce que defunct de bonne \& immortelle memoyre monsieur Budé en a dict en une epistre cy apres inserée, traduicte de latin en nostre langue par laquelle on peult congnoistre combien iceluy tant pur \& excellent jugement d'homme a estimé ce petit livre digne d'estre leu $[\ldots], 62$

En s'alignant ainsi sur la position de l'auteur du De Asse, et en lui donnant la priorité (et même l'exclusivité) sur les autres textes accompagnant 1'Utopie, Le Blond fait siennes les idées du grand humaniste français. Si l'on revient au contenu de cette lettre, on comprend mieux pourquoi Le Blond agit de la sorte.

61 «Les hommes doibvent estre attirez a religion par louenge », « Femmes eslues a la dignité de la pbrestise », « Excommunication des Utopiens》, « Comme sont leurs eglises

62 Jean LE BLOND, dans Th. More, La description de l'isle d'Utopie, op. cit., f. O II.
Ainsi, Budé écrit que, pris par sa lecture, et concentré sur les mœurs et les institutions des Utopiens, il interrompt et délaisse

le soulcy pourchas de mes affaires domesticques voyant que l'art \& industrie economicque qui ne tend sinon que a augmenter le revenu est chose vaine. ${ }^{63}$

Commence alors la première véritable interprétation de l'Utopie, dans laquelle Budé va pousser plus loin une réflexion que More ne fait qu'esquisser dans son ouvrage. Budé s'en prend aux hommes de loi qui se sont spécialisés dans le détournement de celle-ci au profit des puissants, pour leur permettre d'accumuler toujours plus de richesses. Cette conception négative de la réalité est à rapprocher de la critique de la société par More au livre I et de la lettre de Busleiden qui accompagne la première édition de l'Utopie. La critique sociale qui en découle est fondée sur le fait que la nature humaine n'est pas parfaite. Budé donne une analyse du droit, qui lui sert d'exemple pour sa critique, puis rapproche pour la première fois les Utopiens et les premiers chrétiens grâce à l'exemple d'Ananie. ${ }^{64}$ Budé tire l'Utopie du côté de l'Évangile, et réciproquement. Il continue de la sorte :

Pourtant l'Ile d'Utopie que j'entens aussi estre appellée Utopodie, ${ }^{65}$ a d'une merveil Pourtant lile diestiennes, \& mesmes leuse araye sapience \& en publicq \& en privé qu'elle a gardé jsuques a huy sans y rien

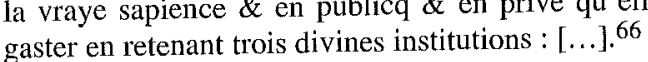

On remarque que Budé attribue explicitement le qualificatif de «chrestiennes » aux coutumes des Utopiens. La confusion entre Utopie et Paradis chrétien revient de même dans le «Dixain du translateur à la louenge de la saincte vie des Utopiens » :

Je dys cecy car quand bien on lyra,

Les sainctes meurs d'Utopie, on dira:

C'est paradis au prix du lieu où sommes ;

Touchant les gens, on les estimera

Estre espritz sainctz plus tost que mortelz hommes. ${ }^{67}$

Le Blond, par ce dizain, rattache 1'Utopie à une certaine forme de sainteté, malgré la différence évidente qu'il remarque entre le culte entre les chrétiens et celui des Utopiens. La lecture qu'il en fait est donc influencée par l'interprétation

63 «Guillaume Budé à Thomas Lupset Angloys », dans Ibid., non paginé

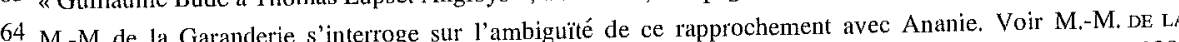
64 M.-M. de la Garre

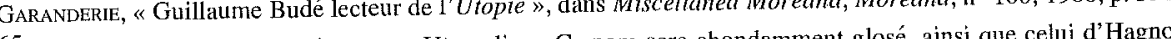
65 Le Blond traduit « Udepotia » par « Utopodie ». Ce nom sera abondamment glose, ansi que celui d Hagnopolis. Pour en savoir plus, voir les références suivantes : pour" «Udepotla», G. HonkE, «Die Rezeption der

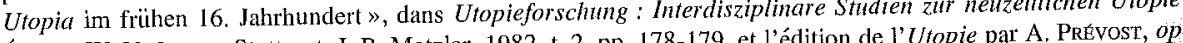

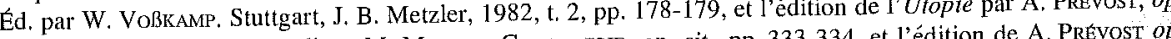

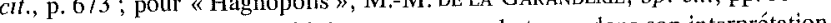
cit., p. 325, ainsi que les multiples occurrences du terme dans son interprétation.

66 "Guillaume Budé à Thomas Lupset Angloys», op. cit., non paginé.

67 Jean Le Blond, «Dixain du translateur à la louenge de la saincte vie des Utopiens», dans Th. More, $L a$ description de l'isle d'Utopie, op. cit., non paginé. 
de Budé, et penche vers une description - comme le titre de sa traduction le laisse voir - d'une sorte de paradis chrétien. Mais ce n'est qu'un des aspects sous lesquels Le Blond a envisagé l'Utopie.

En effet, pour revenir à la lettre de Budé, qui cite «trois divines institutions ", à savoir l' " esqualité des biens \& maulx entre citoyens », un « constant \& perseverent amour de paix \& tranquilité » et un «mespris dor \& dargent », on voit qu'il s'agit en fait de souhaits concernant la perfectibilité de l'homme. Ces souhaits pourraient se comprendre comme une volonté d'améliorer des tares traînées par l'homme depuis le péché originel, ainsi que le défend Honke. ${ }^{68}$ Mais il faut aller plus loin, et voir en l'Utopie un moyen de dépasser ces tares. Les dernières lignes de la lettre de Budé vont dans ce sens :

Certes lhistoire de ceste Isle de nostre aage \& a noz successeurs comme une pepiniere delegantes \& utiles institutions desquelles ilz pourront tyrer meurs pour retenir \& accorder chascun en sa cité. 69

Elles reprennent l'idée que l'on retrouve à la fin de l'Utopie :

Je suis joyeux que ceste maniere de republicque laquelle je desire a toutes aultres nations \& est escheue aux Utopiens, qui ont ensuivy si bonne forme de vivre, par laquelle ilz ont si bien fondé leur republicque, $\&$ si heureusement, qu'elle sera perdurable, ainsi qu'en peuvent deviner les hommes par conjecture humaine. ${ }^{70}$

Cette république peut servir de modèle politique. Le Blond l'a très bien compris, et il ne conclut pas son avertissement au lecteur autrement, en expliquant qu'il espère avoir bien fait de traduire ce livre,

en quoy duquel j'ay pretendu, comme de tout aultre mien labeur, faire chose qui soit a l'utilité \& proffit de la republicque. A dieu. ${ }^{71}$

L'Utopie est donc une étape cohérente dans les démarches linguistique, religieuse et politique de Le Blond. Euvre riche et aux problématiques multiples, elle correspond à une sorte de rédemption de l'homme par l'homme. En effet, si la perfectibilité de l'homme dénoncée par Budé est bien liée au péché originel, l'Utopie se propose à l'homme comme un instrument de réflexion pour accomplir cette tâche, ce perfectionnement. En poussant plus loin le raisonnement, on peut même affirmer que la traduction de l'Utopie constitue un aboutissement pour Le Blond, mais aussi une solution de continuité dans son œuvre : après celle-ci, il se tournera en effet vers l'histoire, ${ }^{72}$ avec Les Chroniques de Jean Carion et l'Histoire de Neustrie.

\footnotetext{
68 G. HoNkE, op. cit., pp. 167-182.

69 «Guillaume Budé à Thomas Lupset Angloys », op, cit., non paginé.

70 Th. More, La description de l'isle d'Utopie, op. cit., f. 104v".

71 Jean LE BLOND, dans Ibid, f. O III.

72 Même si cette histoire, nous l'avons vu, garde des ambitions politiques.
} 